J. Gynäkol. Endokrinol. CH 2021 · 24:104-107 https://doi.org/10.1007/s41975-021-00204-0 Angenommen: 26. Mai 2021

Online publiziert: 16. Juni 2021

(c) Der/die Autor(en) 2021

\title{
Sabrina Baumgartner
}

Frauenklinik, Universitätsspital Zürich, Zürich, Schweiz

\section{Älterwerden aus der Perspektive der Sportlerin}

Dass regelmässige körperliche Aktivität ein Allerweltsmittel ist zur Prävention der häufigsten Volkserkrankungen wie Herz-Kreislauf-Erkrankungen, Diabetes mellitus Typ 2, Adipositas, Krebserkrankungen (insbesondere Darm- und Brustkrebs) und Osteoporose, ist kein Geheimnis. Dennoch hat man sich mit dem Sporttreiben in den jungen Jahren noch kein Ticket für die bedingungslose Gesundheit gesichert. Zwischenzeitlich haben viele Arbeiten gezeigt, dass regelmässige physische Aktivität den Alterungsprozess verlangsamt. Ganz nach dem Motto: „Wer rastet, rostet“. Dass sportliche Tätigkeit mit zunehmendem Alter nicht mit derselben Leichtigkeit wie in jungen Jahren verbunden ist, liegt auf der Hand. Manch einer mag sich fragen: Wie erleben Sportlerinnen das Älterwerden und welche Rolle nimmt Sport beim Älterwerden ein? Wir haben dazu sowohl die ehemalige Weltklasseläuferin Sandra Gasser (• Abb. 1) als auch die noch aktive und international erfolgreiche 79-jährige Mehrkämpferin in der Kategorie Masters Marianne Maier (- Abb. 2) befragt.

Sandra Gasser, die ehemalige Schweizer Weltklassemittelstreckenläuferin ( $800 \mathrm{~m}$ und $1500 \mathrm{~m}$ ), hat uns Einblick gegeben in ihren Lebensabschnitt nach der Sportkarriere. Zu den sportlichen Höhepunkten von Sandra Gasser gehören der 3. Platz bei den Europameisterschaften über $1500 \mathrm{~m}$ (1990) und eine Bestzeit über $800 \mathrm{~m}$ von 1:58:90, mit welcher sie über 28 Jahre den Schweizer Rekord innehatte, bis dieser 2015 von Selina Büchel geknackt wurde. Der Entschluss zum Rücktritt vom Spitzensport wurde ihr damals aufgrund einer Diskushernie ein wenig erleichtert. Für sie war von Beginn an klar, dass ein kompromissloser
Schlussstrich unter die Sportlervergangenheit gezogen werden muss, um sich erfolgreich neu orientieren zu können. Dass Sandra Gasser zur regelmässigen körperlichen Aktivität auch heute noch keinen „inneren Schweinehund“ überwinden muss, verrät sie uns, indem sie von „Trainings streichen“ spricht. Sie reduzierte nach dem Karriereende das Trainingspensum schrittweise, um mehr Raum für Neues zu schaffen, aber zugleich den positiven Nebeneffekt der regelmässigen körperlichen Betätigung beizubehalten. Wer jetzt denkt, dass Sandra Gasser ihre Laufschuhe mit 59 Jahren nicht mehr regelmässig schnürt, täuscht sich. Tatsächlich fühlt es sich wohl aus der Perspektive einer Spitzensportlerin so an. Sie laufe zurzeit 4-mal wöchentlich $40 \mathrm{~min}$ in gemütlichem Tempo „praktisch nichts“, fügt sie lachend an. Mit diesem Trainingspensum deckt sie just die Empfehlungen der WHO, welche mindestens 150 min moderate körperliche Aktivität oder $85 \mathrm{~min}$ hohe Intensität wöchentlich empfiehlt. Mit den $4 \times 40$ minütigen Laufeinheiten kann sie sich ebenso zu denjenigen zählen, die gemäss einer im Jahr 2019 publizierten prospektiven, randomisiert, kontrollierten Studie effektives und kostenfreies AntiAging betreiben [1]. So konnte gezeigt werden, dass regelmässiges Ausdaueroder Intervalltraining (3-mal $45 \mathrm{~min}$ pro Woche) die enzymatische Telomeraseaktivität aufrechterhält. Damit werden die repetitiven Nukleotidsequenzen (Telomere) am Ende der Chromosomen vor der Verkürzung bewahrt, was den Alterungsprozess der Körperzellen verlangsamt. In Anbetracht der körperlichen Leistungsfähigkeit nimmt Sandra Gasser das Älterwerden durch abnehmende Schnellkraft und vermindertes 


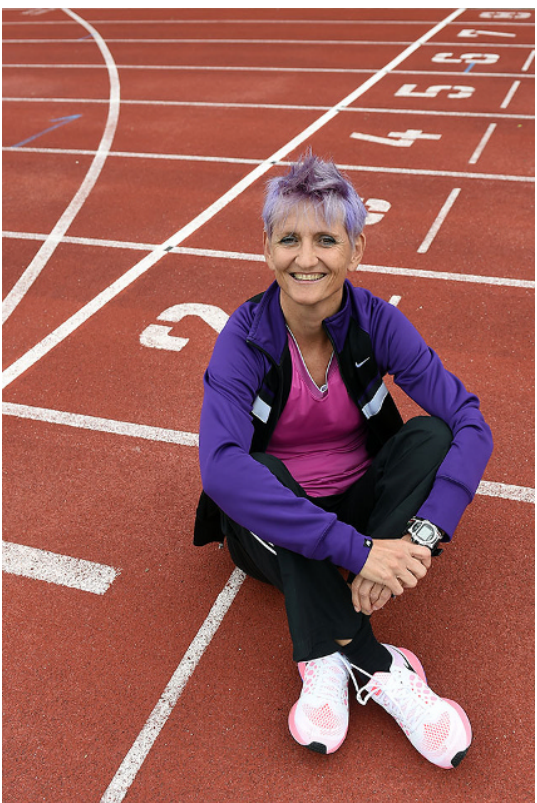

Abb. 1 ॥ Sandra Gasser, ehemalige WeltklasseMittelstreckenläuferin. (@ Andreas Blatter [Berner Zeitung]. Diese Abbildung fällt nicht unter die Creative Commons CC BY-Lizenz dieser Publikation)

Koordinationsvermögen wahr. Die regelmässige körperliche Aktivität helfe ihr, dieser Entwicklung entgegenzuwirken. Aus sportwissenschaftlicher Sicht macht Sandra Gasser damit intuitiv vieles richtig. Die Abnahme der Schnellkraft im Alter wird durch den Verlust der F-Muskelfasern (Typ 2, „fast-twitch“) erklärt [2]. Im Gegensatz zu den S-Muskelfasern (Typ 1, „slow-twitch“) nimmt die Regenerationsfähigkeit der F-Fasern im Alter in grösserem Ausmass ab. Die Diskrepanz der unterschiedlichen Regenerationsfähigkeit abhängig vom Muskelfasertyp kommt durch die akzentuierte Apoptose der F-Faser-spezifischen Satellitenzellen zustande. Die Satellitenzellen fungieren als „Muskelstammzellen" und differenzieren bei anabolischen Stimuli zu neuen Myonuklei, welche mit vorbestehenden Fasern fusionieren oder neue Fasern generieren [3, 4]. Regelmässiges Krafttraining wirkt der Apoptose der Satellitenzellen entgegen und damit einer alters- und inaktivitätsgeschuldeten Muskelatrophie. Folglich nehmen Schnellkraftleistungen im Alter schneller ab als Kraft- und Ausdauerleistungen. Sandra Gasser vermutet, dass eine ehemalige Sportlerin

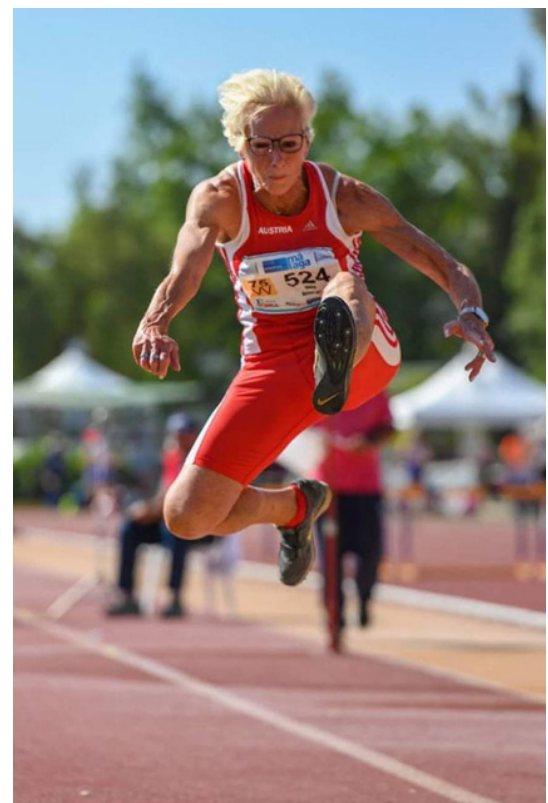

Abb. 2 A Marianne Maier, Masterathletin Leichtathletik 7-Kampf. (Mit freundlicher Genehmigung von Marianne Maier, Diese Abbildung fällt nicht unter die Creative Commons CC BY-Lizenz dieser Publikation)

gezwungenermassen intensiver mit dem Altern konfrontiert wird. Als Sportlerin hätte sie sich über die erbrachte Leistung, aber auch über den durchtrainierten Körper definiert, der ihr gefallen habe. Die altersbedingte Differenz zum Ist-Zustand sei bei ehemaligen Spitzensportlern grösser und werde einem täglich durch den Spiegel vor die Augen geführt. Die Herausforderung bestehe darin, ein neues Gleichgewicht zu finden zwischen Akzeptanz der altersbedingten Veränderungen und dem Aufrechterhalten der körperlichen Betätigung, welche durchaus auch herausfordernd sein darf. Sandra Gasser erwähnt in diesem Kontext eine grosse innere Zufriedenheit über das Geleistete als auch Dankbarkeit für den gesunden Körper. Auf die Frage, welche Ansprüche ihr durch den Sport an ihren Körper erhalten geblieben sind, entgegnet Sandra Gasser prompt: „Die Körperhaltung und agil zu bleiben.“ Das sind Eigenschaften, die Sandra Gasser auch auf ihr Leben übertragen hat: Ein starkes Rückgrat im Sinne eines gesunden Selbstbewusstseins hilft ihr im Alltag noch heute, mit Ruhe, Stetigkeit und Zuversicht auf ein Ziel hinzuarbeiten. Eigenschaften, die sie als beherzte
Mittelstreckentrainerin ihren Athleten noch heute auf den Weg mitgibt.

Wieso Leistungssport auch in der zweiten Lebenshälfte als „Jungbrunnen“ taugt, zeigt uns die gebürtige Österreicherin Marianne Maier (79-jährig) aus St. Margrethen (SG) beispiellos auf. Marianne Maier darf sich zurzeit Europas beste Mehrkämpferin in der Kategorie Masters nennen. Zur Kategorie Masters gehört man ab dem 35. Lebensjahr, wobei man fortan in 5-Jahres-Schritten in die nächste Masterkategorie aufsteigt (z. B. Kategorie 35-39, 40-45). Kurzum hat Marianne Maier seit ihrem ersten Einsatz als Masterleichtathletin, dazumal noch 45-jährig, bis und mit heute über 50 Medaillen an Welt- und 45 Medaillen an Europameisterschaften ergattert. Auf den ersten Blick mag es so wirken, als ob die sportliche Aktivität von Marianne Maier einen Einzelfall darstellt. In Tat und Wahrheit entspricht sie dem aktuellen Trend mit der zunehmenden Partizipation von Masterathleten und -athletinnen an ausdauerbetonten Sportveranstaltungen in den vergangenen drei Dekaden [5]. So waren beispielsweise am BostonMarathon $2016 \sim 60 \%$ über 40 Jahre alt [6]. Mit dem regelmässigen Sporttreiben hat Marianne Maier erst mit 40 Jahren begonnen, als mehr Zeit zur Verfügung stand. Jeder, der bereits einen Siebenkampf bestritten hat, weiss, welche Technikarbeit allein hinter der Tatsache verborgen liegt, einen Siebenkampf absolvieren zu können. Der „Siebenkampf“ ist ein zweitägiger Wettbewerb in der Leichtathletik - bestehend aus den Disziplinen $100 \mathrm{~m}$ Hürden, Hochsprung, Kugelstossen, 200-Meter-Lauf, Weitsprung, Speerwurf und 800-Meter-Lauf. Dass man durch tägliches Üben auch mit zunehmendem Alter Fortschritte erzielen kann, beweist Marianne Maier anhand ihres Palmarès. Die Motive, welche Masterathleten dazu bewegen, weiterzutrainieren, sind: Vergnügen, Wettkämpfe und das Aufrechterhalten der Fitness und Gesundheit sowie der sozialen Kontakte [7]. Worin besteht bei Marianne Maier trotz der 79 Jahre die Motivation, sich nicht nur regelmässig körperlich zu betätigen, sondern auch fokussiert auf ein Ziel hinzuarbeiten? Anhand der Erläuterung von Marian- 
ne Maier war die Bekanntschaft mit der Leichtathletik damals eine Art „Liebe auf den ersten Blick“. Der Siebenkampf habe in ihr eine Faszination ausgelöst, welcher sie bis heute leidenschaftlich nachgeht. Gemäss Marianne Maier brauche es im Alter deutlich mehr Eigeninitiative, diesen „inneren Schweinehund“ täglich von Neuem zu bezwingen. Der Berg der Überwindung werde stets höher aufgrund der zunehmenden körperlichen Müdigkeit bei zeitgleich abnehmendem Tatendrang, beobachtet Maier. Inwiefern profitiert Marianne Maier von der regelmässigen sportlichen Aktivität abgesehen von den sportlichen Erfolgen? Durch das regelmässige Sporttreiben hätte sie verglichen mit ihren Altersgenossen kaum Wechseljahrbeschwerden verspürt. Das regelmässige Training verbessere ihr Gleichgewicht, ihre Beweglichkeit und ihre Koordination. Diese Eigenschaften generieren ihr mehr Selbständigkeit im Alltag. Wird Marianne Maier nach ihren Schwächen gefragt, entgegnet sie wortwörtlich mit Schalk: „Für mich ist es ein Problem, wenn ich langsam laufen muss." Diese vermeintliche Schwäche hat ihr wohl zu ihrem sportlichen Erfolg und zur heutigen körperlichen Leistungsfähigkeit verholfen. Die Motivation von Marianne Maier ist beeindruckend und ansteckend. Motivation entstammt dem lateinischen Wort „movere“. Marianne Maier will ungeachtet ihres Alters in Bewegung bleiben. Ganz nach dem Motto: „Nur wer sich in Bewegung setzt, kann etwas bewegen.“

\section{Korrespondenzadresse}

\section{Dr. med. Sabrina Baumgartner}

Frauenklinik, Universitätsspital Zürich

Zürich, Schweiz

sabrina.baumgartner@usz.ch

Funding. Open access funding provided by University of Zurich

\section{Einhaltung ethischer Richtlinien}

Interessenkonflikt. S. Baumgartner gibt an, dass kein Interessenkonflikt besteht.

Für diesen Beitrag wurden von der Autorin keine Studien an Menschen oder Tieren durchgeführt. Für die aufgeführten Studien gelten die jeweils dort angegebenen ethischen Richtlinien.
Open Access. Dieser Artikel wird unter der Creative Commons Namensnennung 4.0 International Lizenz veröffentlicht, welche die Nutzung, Vervielfältigung Bearbeitung, Verbreitung und Wiedergabe in jeglichem Medium und Format erlaubt, sofern Sie den/die ursprünglichen Autor(en) und die Quelle ordnungsgemäß nennen, einen Link zur Creative Commons Lizenz beifügen und angeben, ob Änderungen vorgenommen wurden.

Die in diesem Artikel enthaltenen Bilder und sonstiges Drittmaterial unterliegen ebenfalls der genannten Creative Commons Lizenz, sofern sich aus der Abbildungslegende nichts anderes ergibt. Sofern das betreffende Material nicht unter der genannten Creative Commons Lizenz steht und die betreffende Handlung nicht nach gesetzlichen Vorschriften erlaubt ist, ist für die oben aufgeführten Weiterverwendungen des Materials die Einwilligung des jeweiligen Rechteinhabers einzuholen.

Weitere Details zur Lizenz entnehmen Sie bitte der Lizenzinformation auf http://creativecommons.org/ licenses/by/4.0/deed.de.

\section{Literatur}

1. Werner CM, Hecksteden A, Morsch A, Zundler J, Wegmann M, Kratzsch J, Thiery J, Hohl M, Bittenbring JT, Neumann F, Böhm M, Meyer T, Laufs U (2019) Differential effects of endurance, interval, and resistance training on telomerase activity and telomere length in a randomized, controlled study. Eur Heart J 40(1):34-46. https:// doi.org/10.1093/eurheartj/ehy585

2. Coggan A, Spina R, Rogers M et al (1990) Histochemical and biochemical characteristics of skeletal muscle in master athletes. J Appl Physiol 68:1896-1901

3. Larsson L, Sjodin B, Karlsson J (1978) Histochemical and biochemical changes in human skeletal muscle with age in sedentary males, age 22-65 years. Acta Physiol Scand 103:31-39

4. Verdijk LB, Koopman R, Schaart G, Meijer K, Savelberg HH, van Loon LJ (2007) Satellite cell content is specifically reduced in type II skeletal muscle fibers in the elderly. Am J Physiol Endocrinol Metab 292(1):E151-E157. https://doi. org/10.1152/ajpendo.00278.2006

5. Jokl P, Sethi PM, Cooper AJ (2004) Master's performance in the New York City Marathon 1983-1999. Br J Sports Med 38(4):408-412. https://doi.org/10.1136/bjsm.2002.003566

6. Tanaka H (2017) Aging of competitive athletes. Gerontology 63(5):488-494. https://doi.org/10. $1159 / 000477722$

7. Medic N (2010) Motivation and sport commitment in Master Sport. ISBN 978-0-415-47657-7

Hinweis des Verlags. Der Verlag bleibt in Hinblick auf geografische Zuordnungen und Gebietsbezeichnungen in veröffentlichten Karten und Institutsadressen neutral.

\section{Telemedizin}

Marx, Gernot, Rossaint, Rolf, Marx, Nikolaus (Hrsg.)

2021, XVI, 502 S. 98 Abb., 79,99 EUR ISBN 978-3-662-60610-0

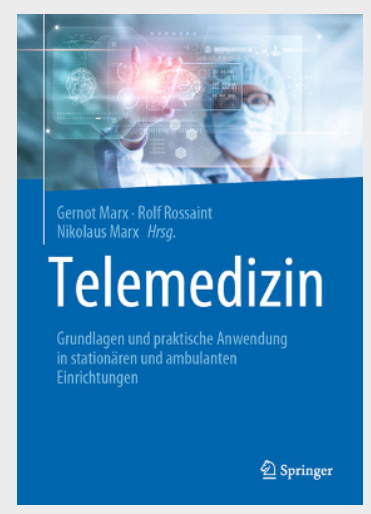

Grundlagen und praktische Anwendung in stationären und ambulanten Einrichtungen:

- Chancen, Risiken und rechtliche Besonderheiten der Telemedizin

- Mit zahlreichen Anwendungsbeispielen

- Mit Blick in die EU und Nachbarländer

Das Buch gibt einen umfassenden Überblick über den aktuellen Stand und die Entwicklungen im Bereich der Telemedizin in Deutschland. Das Herausgeberteam aus einem der führenden Telemedizinzentren Deutschlands und ein interdisziplinäres Autorenteam liefern wesentliche Grundlagen sowie zahlreiche Anwendungsbeispiele aus dem stationären und ambulanten Bereich. Zudem werden Chancen und Risiken der Telemedizin erörtert, so dass der Leser gut für die Zukunft in Klinik oder Praxis gerüstet ist. Ein Grundlagen- und Nachschlagewerk für alle Ärzte, die sich für digitale Zukunft im Gesundheitswesen wappnen und ihre Patienten optimal versorgen möchten. 
Hier steht eine Anzeige.

\section{曾 Springer}

